\title{
The aetiology of acute community acquired bacterial meningitis in children and adults in Maputo, Mozambique
}

\author{
Tomás F. Zimba ${ }^{1,2,3,4}$, David T. Nota ${ }^{1}$, José C. Langa ${ }^{3}$, Leonel G. S. Monteiro ${ }^{3}$ and Yacoob M. \\ Coovadia $^{5}$
}

${ }^{1}$ Laboratório de Microbiologia and ${ }^{2}$ Departamento de Medicina at the Hospital Central de Maputo; ${ }^{3}$ Laboratório Referência de Bacteriologia at the Instituto Nacional de Saúde; ${ }^{4}$ Instituto Superior de Ciências de Saúde; ${ }^{5}$ Department of Medical Microbioloy, Nelson R Mandela School of Medicine and NHLS Laboratory Services, Kwa Zulu Natal

\begin{abstract}
Background: Community-acquired acute bacterial meningitis is a life-threatening infection and many outbreaks have been reported all around the world.

Methodology: We analysed 330 cerebrospinal fluid (CSF) samples received over a period of eight months from patients older than one month. Microscopy, cultures, identification, and antigen detection were performed for the positive samples.

Results: Neisseria meningitidis, Streptococcus pneumoniae and Haemophilus influenzae type b were isolated in 5.4\%, $4.8 \%$, and $3.6 \%$ of CSF samples respectively.

Conclusions: Our findings indicate that $N$. meningitidis is the most common cause of acute bacterial meningitis in Mozambique and that $H$. influenzae type $\mathrm{b}$ was isolated only from children aged younger than six years. This is the first study to provide data on the aetiological agents of acute bacterial meningitis in children and adults in Mozambique.
\end{abstract}

Keywords: Community-acquired meningitis, Mozambique

J Infect Dev Ctries 2009; 3(9):723-726.

Received 28 November 2008 - Accepted 21 July 2009

Copyright () 2009 Zimba et al. This is an open-access article distributed under the Creative Commons Attribution License, which permits unrestricted use, distribution, and reproduction in any medium, provided the original work is properly cited.

\section{Introduction}

Community-acquired acute bacterial meningitis is a severe and life-threatening infection if not diagnosed early and treated appropriately [1]. Neisseria meningitides, Haemophilus influenza type b and Streptococcus pneumoniae are the most common causes of acute bacterial meningitis in developing countries [2]. Bacterial meningitis caused by $H$. influenza type $\mathrm{b}$ and $S$. pneumoniae have been declining in the developed countries as result of the introduction of specific vaccines [1].

N. meningitidis, a Gram-negative diplococcus, is a major cause of community-acquired acute bacterial meningitis worldwide $[1,3,4,5]$. This bacterium is divided into 13 serogroups based on the antigenicity of the polysaccharide capsule 6]. Serogroups A, B, C, $\mathrm{W} 135, \mathrm{Y}$, and $\mathrm{X}$ are associated with seasonal outbreaks [3]. Genetic recombination has been reported to play a role in the emergence of these outbreaks [1]. In the African meningitis belt, epidemic meningococcal infections caused an estimated 700,000 cases with a mortality rate of $10 \%$ in the last 10 years [7]. N. meningitidis causes bacterial meningitis in $80-95 \%$ of cases during epidemic periods and up to $50 \%$ in non-epidemic situations in the above mentioned African area $[8,9,10]$.

$H$. influenza type $\mathrm{b}$ and $S$. pneumoniae are commonly isolated in patients presenting with meningitis in countries where the new conjugated vaccines are not in use [1]. S. pneumoniae is a major cause of otitis media, pneumonia, and meningitis in all age groups [11], while $H$. influenza type $\mathrm{b}$ is isolated mainly from patients younger than 6 years presenting with the same diseases [12]. These two pathogens have assumed an even more important role as causative agents of serious infections in patients infected with human immunodeficiency virus (HIV) [13].

In the absence of reliable data on the prevalence of $S$. pneumoniae and $H$. influenza type b infections in many developing countries, it is difficult for National Health Programs to motivate authorities to 
Table 1

Aetiology of acute community bacterial meningitis at Maputo Central Hospital

\begin{tabular}{lcc}
\hline \multicolumn{1}{c}{ Causative agent } & Number & Percentage \\
\hline Neisseria meningitidis & 18 & 5.4 \\
Streptococcus pneumoniae & 16 & 4.8 \\
Haemophilus influenzae type b & 12 & 3.6 \\
Negatives & 284 & 86.2 \\
\hline
\end{tabular}

implement vaccines against these two important pathogens. In Mozambique, very little is known about the extent of burden of disease attributed to community-acquired acute bacterial meningitis $[2,19]$. The main objective of this study, therefore, was to determine the aetiology of community-acquired acute bacterial meningitis in patients older than one month presenting to the Maputo Central Hospital.

\section{Materials and Methods}

Cerebrospinal fluid (CSF) samples were received from patients older than one month in the microbiology laboratory at the Maputo Central Hospital between August 2007 and March 2008. The samples were centrifuged at $3,000 \mathrm{rpm}$ for 10 minutes. The supernatant was aseptically removed, and a Gram stain performed on the deposit. A drop of the remaining CSF was inoculated onto blood and chocolate agar. The two plates were incubated in a candle jar at $35^{\circ} \mathrm{C}$ for $24-48$ hours.

A Gram stain was performed from of all positive cultures. An oxidase test was performed on all isolates of Gram-negative diplococci, which were then further identified as pathogenic Neisseria by determining the absence of growth on a chocolate agar plate incubated at room temperature. Gramnegative coccobacilli resembling Haemophilus were tested for $\mathrm{X}, \mathrm{Y}$ and $\mathrm{XY}$ factor requirement on nutrient agar plates incubated at $37^{\circ} \mathrm{C}$ in candle jars. $S$. pneumoniae was identified on the basis of the Gram stain appearance and colony morphology; susceptibility to optochin was tested on subculture in blood agar plate placed with this disc on the initial streak.

The Wellcogen Bacterial Antigen Kit (Remel Europe Ltd) for $N$. meningitidis A, C,Y, W135 and $\mathrm{B} /$ E. coli $\mathrm{K} 1, H$. influenzae type $\mathrm{b}$, Streptococcus group B, and S. pneumoniae was used to confirm the identification of isolates of Gram-positive diplococci,
Gram-negative diplococci and Gram-negative coccobacilli.

\section{Results and Discussion}

A total of 330 CSF samples were processed in the microbiology laboratory during the period August 2007 to March 2008. These samples were received from patients in the Pediatrics, Medicine, Intensive Care Unit, and Gynecology departments of Maputo Central Hospital. The patients' ages varied from one month to 68 years. Of the $330 \mathrm{CSF}$ samples received, $46(13.8 \%)$ were positive on culture (Table 1$). H$. influenza type $\mathrm{b}$ was cultured from 12 (3.6\%) samples, S. pneumoniae was cultured from 16 (4.8\%), and $N$. meningitidis was cultured from 18 (5.4\%). With the exception of two patients for whom the age was not available, $H$. influenzae type $\mathrm{b}$ isolates were recovered only from children younger than 6 years $(P$ value of 0.0004). S. pneumoniae was evenly distributed in all age groups. $N$. meningitidis, however, was more commonly isolated from patients aged one to 20 years ( $P$ value is 0.007 ) (Table 2$)$. The age was not indicated for 55 patients, two of whom were infected with $H$. influenzae type b meningitis.

The diagnosis of acute bacterial meningitis in many developing countries is usually based on clinical and basic laboratory criteria (i.e., CSF microscopy and chemistry) because of the lack of qualified personnel and minimally equipped microbiology laboratories. It is therefore not surprising that information on the aetiology of acute bacterial meningitis is not readily available in such countries including Mozambique. With the introduction of highly effective conjugate polysaccharide vaccines against $H$. influenzae type $\mathrm{b}$ and $S$. pneumoniae, it is essential that such information be available in developing countries [1].

In the present study, $13.9 \%$ of CSF samples were culture positive for aetiological agents of acute bacterial meningitis. This observation is in keeping 
Table 2

Relationship between patient age and causative agents of bacterial meningitis at Maputo

\begin{tabular}{cccccccc}
\hline Patient age & H. influenzae type b & \multicolumn{2}{l}{ S. pneumoniae } & N. meningitis & $\begin{array}{c}\text { Total of } \\
\text { patient }\end{array}$ \\
\hline Years & $\mathrm{n}$ & $\%$ & $\mathrm{n}$ & $\%$ & $\mathrm{n}$ & $\%$ & $\mathrm{n}$ \\
\hline$<1$ & 7 & 2.1 & 4 & 1.2 & 2 & 0.6 & 78 \\
1 to 5 & 3 & 0.9 & 1 & 0.3 & 4 & 1.2 & 52 \\
6 to 10 & 0 & 0 & 1 & 0.3 & 6 & 1.8 & 17 \\
11 to 20 & 0 & 0 & 1 & 0.3 & 2 & 0.6 & 20 \\
$>20$ & 0 & 0 & 2 & 0.6 & 1 & 0.3 & 108 \\
No age & 2 & 0.6 & 7 & 2.1 & 3 & 0.9 & 55 \\
Total & 12 & 3.6 & 16 & 4.8 & 18 & 5.4 & 330 \\
\hline
\end{tabular}

with the findings of the only other study reported form Mozambique from Manhiça district [2], in which $15 \%$ of the patients had culture proven meningitis. However, the latter was performed only in patients aged younger than 15 years living in rural area. The previous study also reported that $S$. pneumoniae, $H$. influenza, and $N$. meningitidis were the most common bacteria isolated from the patients presenting with signs of meningitis [2].

We found $N$. meningitidis to be the commonest isolate, which contrasts with findings of the study reported from Manhiça district of Mozambique, where it was found to be the third commonest cause and was isolated in only $1.6 \%$ of CSF samples [2]. Furthermore, N. meningitidis in the present study was isolated significantly more frequently in the age group one to twenty years.

It has also been reported that more than $50.0 \%$ of cases of meningococcal meningitis occur in subSaharan African countries including Mozambique $[15,16]$, and yet a vaccine against this bacterium is still not part of the routine vaccination program in Mozambique [2].

S. pneumoniae was found to be the second commonest cause of acute bacterial meningitis, accounting for $4.8 \%$ of positive cultures. In contrast, Sigauque et al. [2] reported $6.5 \%$ of meningitis cases caused by this organism, which in their study was found to be the commonest cause in children. $S$. pneumoniae has also been reported to be the commonest cause of acute bacterial meningitis in other published studies from elsewhere in the world, which led to the introduction of conjugated polysaccharide pneumococcal vaccine in many developed countries and resulted in a significant decline of invasive pneumococcal infection $[1,14]$. The pneumococcal serotypes found in Mozambique are currently unknown and need to be urgently addressed in future studies. This information will prove invaluable in determining whether currently available pneumococcal vaccines will adequately cover serotypes prevalent in Mozambique.

In the present study, $H$. influenzae type $\mathrm{b}$ was found in $3.6 \%$ of CSF samples, and all positive cultures were in children younger than six years (Table 2), which is in keeping with other reports $[2,12]$. However, we found a higher incidence in comparison with that reported by Sigauque et al. [2], who found $H$. influenzae in only $2.7 \%$ of CSF samples. Both these studies, however, confirm the continuing importance of $H$. influenzae type $\mathrm{b}$ as a cause of meningitis in children aged younger than six years in Mozambique. The government of Mozambique must consider introducing the conjugated $H$. influenzae type b vaccine as part of the routine vaccination program.

It is well recognized that early and appropriate therapy impacts on morbidity and mortality from acute bacterial meningitis $[17,18]$. A major limitation of this study is the lack of data on antimicrobial resistance of meningeal isolates to commonly used antibiotics in the treatment of meningitis in Mozambique. Currently, penicillin or ampicilin and chloranphenicol are used in the empirical treatment of acute bacterial meningitis in Mozambique. 
In conclusion, we believe that further studies are urgently required not only to determine the antimicrobial resistance profile of meningeal isolates in Mozambique, but to also determine the serotypes of these isolates. Information on serotypes will prove invaluable in determining whether currently available vaccines, particularly those against $S$. pneumoniae and $N$. meningitidis, will provide adequate coverage for the population of Mozambique.

\section{Acknowledgements}

Thanks to the American Society of Microbiology for technical assistance, to Dr João Fumane for logistic support and to Dr John F. Alderete for the review.

\section{References}

1. Harrison LH (2006) Prospects for vaccine prevention of meningococcal infection. Clin Microbiol Rev 19: 142-164.

2. Sigauque B, Roca A, Sanz S, et al. (2008) Acute bacterial meningitis among children, in Manhiça a rural area in Southern Mozambique. Ac Trop 105: 2127.

3. Gagneux SP, Hodgson A, Smith TA, et al. (2002) Prospective study of a serogroup X Neisseria meningitides outbreak in North Ghana. JID 185: 618-626.

4. Norheim G, Rosenquist E, Aseffa A, et al. (2006) Characterization of Neisseria meningitides isolates from recent outbreaks in Ethiopia and comparison with those recovered during the epidemic of 1988 to 1989. J Clin Microbiol 44: 861-871.

5. Tyrrel GJ, Chui L, Johnson M, et al. (2002) Outbreak of Neisseria meningitidis in Edmonton, Alberta, Canada. Emerg Infect Dis 8: 519-521.

6. Peltola H (1983) Meningococcal disease: still with us. Rev Infect Dis 5: 71-91.

7. World Health Organization (2007) Standardized treatment of bacterial meningitis in Africa in epidemic and non epidemic situation. WHO/CDS/EPR 3: 1-27.

8. Nathan N, Borel T, Djibo A, et al. (2005) Ceftriaxone as effective as long-acting chloranfenicol in short-course treatment of meningococcal meningitis during epidemics: a randomized study. Lancet 366: 308-313.

9. Parent Du Chatelet I, Traore Y, Gessner BD, et al. (2005) Bacterial meningitis in Burkina Faso: surveillance using field based polymerase chain reaction testing. CID 40: 17-25.

10. Leimkugel J, Forgor AA, Gagneux S, et al. (2005) An outbreak of serotype 1 Streptococcus pneumoniae meningitis in northern Ghana with features that are characteristic of Neisseria meningitidis meningitis epidemics. JID 192: 192 199.

11. Mokadddas EM, Rotimi VO, and Albert MJ (2008) Implications of Streptococcus pneumoniae penicillin resistance and serotype distribution in Kuwait for disease treatment and prevention. Clin Vaccine Immunol 15: 203-207.

12. Tunkel AR and Scheld WM (2005) Acute Meningitis. In Mandell GL, Bennett LE, Dolin R (eds): Mandell, Douglas, and Bennett's Principles and Practice of Infectious Disease. 6th ed. New York: Churchill-Livingstone 1083-1126.

13. Scarborough M, Gordon SB, Wihtty CJM, et al. (2007) Corticosteroids for bacterial meningitis in Adults in SubSaharan Africa. N Engl J Med 357: 2441-2450.
14. Whitney CG, Farley MM, Hadler J, et al. (2003) Decline in invasive Pneumococcal disease after the introduction of protein-polysaccharide conjugated vaccine. N Engl J Med 348: 1737-1746.

15. Lapeyssonnie L (1963) La méningite cerebro-spinale en Afrique. Bull World Health Organ 28 (Suppl): 3-114.

16. Tikhomirov E (1987) Meningococcal meningitis: global situation and control measures. World Health Stat Q 40: 98109.

17. Goetghebuer T, West TE, Wermenbol V, et al. (2000) Outcome of meningitis caused by Streptococcus pneumoniae and Haemophilus influenzae type b in children in the Gambia. Trop Med Int Health 5: 207-213.

18. Saha SK, Baqui AH, Darmstdt GL, et al. (2005) Invasive Haemophilus influenzae type $\mathrm{b}$ disease in Bangladesh with increase resistance to antibiotics. J Pediatr 146: 227-233.

19. Valles X, Flannery B, Roca A, et al. (2006) Serotype distribution and antibiotic susceptibility of invasive and nasopharyngeal isolates of Streptococcus pneumoniae among children in rural Mozambique. Trop Med Inter Health II: 356366.

\section{Corresponding Authors}

Yacoob M. Coovadia and Tomás F. Zimba

Nelson R Mandela Medical School

Department of Medical Microbiology

NHLS Laboratory Services, KZN, Private Bag - 7, Congella - 4013.

Phone: +27 312402787.

Fax: +27 312402786 .

Email: coovadia@ukzn.ac.za

Conflict of Interest: No conflict of interest is declared. 